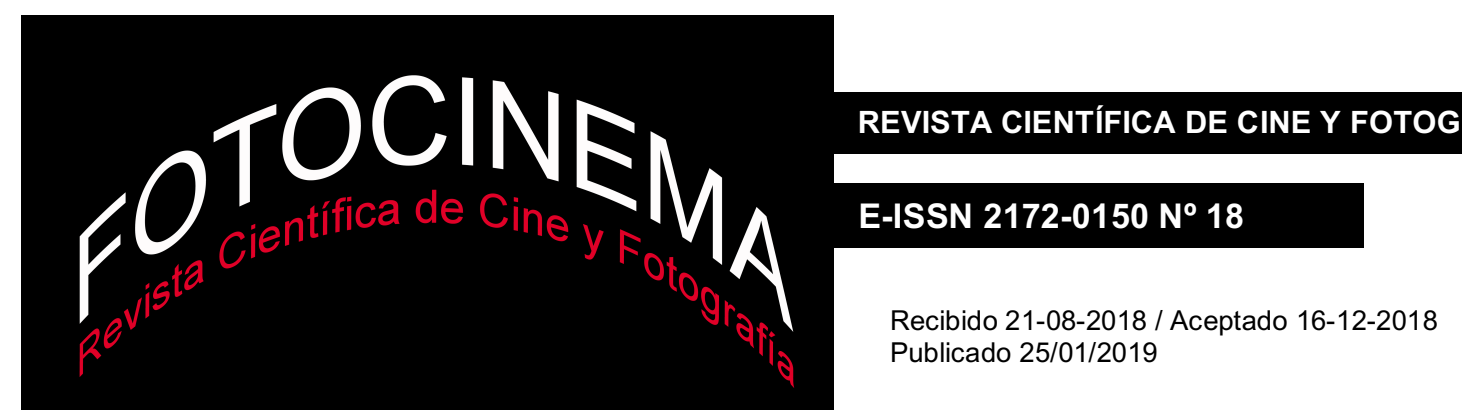

\title{
Metáforas sobre el tiempo y la percepción. Un estudio a propósito de La llegada de Villeneuve
}

\section{Metaphors about time and perception. A study about Villeneuve's movie: Arrival}

\author{
Deborah Rodríguez R. \\ Universidad de Navarra, España. \\ rrdeborah559@gmail.com
}

\section{Resumen:}

El cine se ha convertido en un campo de experimentación y difusión para la filosofía. Es posible reconocer cuestiones centrales para el pensar filosófico que atraviesan las más diversas historias. El género de ciencia ficción se ha vuelto especialmente propicio para esta tarea, dando cabida a escenarios que llevan hasta los límites de lo imaginable el compromiso con ciertos postulados filosóficos. Este es el caso de La Llegada (Arrival), una película de Denis Villeneuve estrenada en 2016. Allí se narra una historia sobre extraterrestres, pero, a diferencias de muchas de sus homólogas, no se centra en destrucción y guerra, sino en los principios del relativismo lingüístico. Siguiendo el trabajo de George Lakoff y Mark Johnson se recorre la historia de Louise Banks, llevando la reflexión hasta la experiencia del espectador. El trabajo de Lakoff y Johnson con respecto al estudio de la metáfora sirve de contexto y explicación para una historia centrada en las consecuencias que los lenguajes tienen sobre la percepción humana. $L a$ llegada conduce al espectador hacia una serie de cuestionamientos sobre el idioma extraterrestre y sobre el propio lenguaje.

\begin{abstract}
:
Movies has become a field of philosophy experimentation and diffusion. Is possible to recognize several main philosophy subjects through many narratives. And Sci-Fi movie genre has become especially propitious to do this. Through the Sci-Fi is possible to create an environment (a place and a time) where reach the limits of main postulates of philosophy and its implications. This is the situation in Arrival, the Denis Villenueve's film from 2016. Is an story about aliens but without war and destruction. In The Arrival, the matter is about the principles of linguistic relativism. Following the George Lakoff and Mark Johnson's work through the fiction character Louise Banks and the reflexion about language. The Lakoff and Johnson's work in relationship to the studies of the metaphor set the context and the explanation to this story. This work is focussed on the consequences of language on human perceptions. The consequences of The Arrival movie audience questions about to learn an alien language and about the human language.
\end{abstract}

Palabras clave:

Metáfora; percepción; lenguaje; filosofía; ciencia ficción; tiempo.

Keywords:

Metaphor; Perception; Language; Philosophy; Sci-Fi; Time. 


\section{Introducción'1}

La RAE define palíndromo como una "palabra o frase cuyas letras están dispuestas de tal manera que resulta la misma leída de izquierda a derecha que de derecha a izquierda”. Así, el castellano permite jugar con oraciones como Amar da drama o Dábale arroz a la zorra el abad. Nótese que para encontrar la gracia de los palíndromos es necesario saltarse ciertas reglas, aparentemente inamovibles, del lenguaje: en primer lugar, aceptar que la lectura puede hacerse de derecha a izquierda y no, como se hace comúnmente, de izquierda a derecha; en segundo lugar, la necesidad de ignorar los espacios entre las palabras. Para que un palíndromo lo sea es necesario que se unifique toda la oración como si se tratase de una única palabra, la cual se puede leer en ambos sentidos. Quien haya jugado con palíndromos entiende que, en ocasiones, lo que está primero podría ser también lo último y lo que en un momento significa retroceder en otro puede ser avanzar. Esto es lo que ocurre en la película de Denis Villeneuve del 2016, La Llegada.

Desde la ciencia ficción, este largometraje considera una importante gama de nociones filosóficas: la finitud de la vida humana; la aceptación de la muerta; la noción de felicidad; el concepto de tiempo y, a su vez, la constitución de la identidad a través de él; la memoria; y el determinismo lingüístico. Sin negar los otros, será este último el tema central del presente texto. Se busca investigar las implicaciones del relativismo lingüístico con motivo de la película de Villeneuve. Esto tiene una importante relación con el trabajo de George Lakoff y Mark Johnson, quienes, a través del estudio de la metáfora, actualizan los postulados originales del relativismo lingüístico y subsanan algunas de sus fallas, por ejemplo, Lakoff y Johnson rescatan la relación fisiológica y sensorial dentro de la construcción de una lengua. Así la película funciona como ejemplo por antonomasia y vívida ilustración de algunos de los postulados que estos autores defienden en su libro Metáforas de la vida cotidiana. Así, la reflexión trasciende la ficción y refiere la realidad humana, abarcando una serie de implicaciones mucho más amplia.

\footnotetext{
${ }_{1}^{1}$ Una primera versión de este texto fue presentada en el LV Congreso de Filosofía Joven, celebrado en Murcia (2018) y dio lugar al Trabajo Final de Máster, todavía inédito: Hábitat Metafórico. Un estudio filosófico a partir de Arrival de D. Villeneuve. Agradezco las correcciones y sugerencias de Antonio Blanco Salgueiro y Jaime Nubiola.
} 
El texto se divide en cuatro apartados adicionales a esta breve introducción: (2) El relativismo lingüístico en La Llegada; (3) Lakoff, Johnson y Villeneuve: La Llegada desde un análisis de la metáfora; (4) La experiencia metafórica del espectador; (5) Conclusiones.

\section{El relativismo lingüístico en La Llegada}

Desde los diálogos de Platón, la filosofía se ha apoyado en los géneros literarios para dar cuenta de sus investigaciones. Con la aparición y popularización del cine, este ha pasado a formar parte de los discursos posibles para difundir y explorar distintas teorías. Esto, lejos de degradar la investigación, permite analizar a través de la ficción las consecuencias más extremas o improbables que se derivan del compromiso con ciertas afirmaciones o posturas filosóficas.

Específicamente, "la ciencia ficción sobre el lenguaje y su vínculo con el pensamiento ha explorado hasta el límite y más allá de lo imaginable con respecto a los alienígenas y también con respecto a nosotros mismo. Esos experimentos resultan útiles para poner a prueba nuestras intuiciones o para contemplar posibilidades y descartar algunas por paradójicas o inconsistentes” (Blanco, 2017, p. 55). La Llegada (Arrival, Denis Villeneuve, 2016), cargada de cuestionamientos epistemológicos y lingüísticos, es un ejemplo de esto.

La historia del film está estrechamente vinculada con los postulados del relativismo lingüístico definido por Benjamin Lee Whorf en su libro Lenguaje, pensamiento $y$ realidad. Actualmente estos postulados han quedado completamente descartados, pero las licencias de la ficción permiten considerarlos. Esto, a su vez, permite reconsiderarlos con el propósito de dar luces sobre ideas que sí se encuentran vigentes. Las nociones del relativismo lingüístico trascienden a su tratamiento original, hoy rechazado.

De forma radical, Benjamín Lee Whorf afirmó "que las personas que utilizan gramáticas acusadamente diferentes se ven dirigidas por sus respectivas gramáticas hacia tipos diferentes de observación y hacia evaluaciones diferentes de actos de observación externamente similares; por lo tanto, no son equivalentes como observadores, sino que tienen que llegar a algunos puntos de vista diferentes sobre el mundo" (Whof, 1971, p. 250). Esto, de forma igualmente 
radical, es lo que le ocurre a la protagonista de la película: al aprender una lengua con una estructura gramatical y conceptual totalmente diferentes se modifica drásticamente su percepción de la realidad.

Originalmente, Whorf se preguntaba si "una civilización como la nuestra hubiera sido posible habiendo contado con un tratamiento lingüístico ampliamente diferentes" (Whorf, 1971, p. 177). Con esto se establece un primer cuestionamiento acerca del lenguaje como mediador entre el hombre y su entorno: como constructor y dador de sentido. Entonces será necesario admitir que, como ocurre con los palíndromos, hay nociones que parecen preexistentes e incuestionables, pero que realmente son modificables. El núcleo de los postulados de Whorf propone considerar que el mundo es como es solo porque los hombres son como son (Ibáñez, 2001, p. 52). Aunque el determinismo lingüístico se mantienen hoy de forma menos radical, un compromiso tan férreo como el de Whorf es actualmente rechazado por la lingüística científica.

Así se desarrolla la historia de Louise Banks, la protagonista de La Llegada. El largometraje de ficción del 2016 fue dirigido por el canadiense Denis Villeneuve y escrita por los estadounidenses Eric Heisserer y Ted Chiang. La trama se basa en el cuento del propio Chiang: La historia de tu vida, recogido en el libro homónimo junto con otros relatos breves del autor. La película cuenta con ocho nominaciones a los premios de la Academia de las Artes y las Ciencias Cinematográficas, entre los que destacan las categorías de Mejor película, Mejor director y Mejores efectos sonoros (en esta última se hizo con el galardón), incluidos entre los más de doscientos reconocimiento y nominaciones cinematográficas.

En la narración, tanto del cuento como del film, se relata la historia de Louise Banks, una reconocida lingüista, a quien se le asigna la tarea de comunicarse con los miembros de una especie extraterrestre que ha arribado a la Tierra. Las criaturas del espacio no pretenden atacar a los humanos y no presentan resistencia ante la interacción. Louise pretende averiguar el propósito que los ha traído a la Tierra y, para ello, decide que la mejor manera es encontrar un código que puedan compartir. A medida que la protagonista aprende la lengua extraterrestre empieza a percibir el mundo bajo unas nociones de tiempo y de 
causalidad completamente diferentes. Es por esto que Louise comienza a tener recuerdos del futuro.

La radical diferencia entre ambas lenguas se centra en su escritura. En la historia de Louise, los extraterrestres se expresan a través de una escritura semasiográgica. Esto quiere decir que, en primer lugar, se comunican a través de logogramas - representaciones visuales que no poseen correspondencia con ningún sonido. Por otro lado, estas representaciones son indivisibles, es decir, una misma impresión es una unidad con sentido completo -toda una oración en un solo "golpe de vista" - como los kanjis japoneses o los primeros nueve números arábigos. En el caso de la ficción de Villeneuve, estas inscripciones son circulares, por lo que no poseen un recorrido de lectura definido. A diferencia de las oraciones en las lenguas humanas, no existe una direccionalidad -de izquierda a derecha como en el caso del inglés o del castellano- por lo que no es posible señalar un punto de inicio y otro de final para cada idea. El lenguaje creado para la película se asemeja más a un compendio de señales de tránsito que a un alfabeto occidental.

Cada una de estas características lingüísticas se incorporan a la nueva forma de percepción que adquiere la protagonista: el tiempo entendido como simultáneo, equidistante y unitario. Así, siguiendo a Whorf, como las estructuras lingüísticas extraterrestre no poseen una noción de continuidad o de linealidad, estas criaturas tampoco son capaces de percibir el mundo bajo esos parámetros. Louise pierde la referencia temporal de los lenguajes humanos, por lo que el pasado, el futuro y el presente se perciben como simultáneos. Villeneuve incorpora esto a la narrativa de la película, dando una sensación de presente mosaico, que intriga al espectador.

Pero entender hoy el relativismo lingüístico siguiendo a Whorf es un camino infructuoso, pues pretender que el lenguaje sea el factor único que moldea y compone la realidad resulta exagerado. El principal fallo, como sus lectores y detractores se han encargado de señalar, es la incompatibilidad entre lenguajes: dentro de un determinismo tan estricto sería imposible aprender más de un idioma o traducir. Pero, aunque los postulados de este autor ya no se sigan, estudios posteriores han rescatado los fundamentos del relativismo lingüístico y los han combinado con estudios culturales y sociales, de modo que, aun 
rechazando a Whorf, no se niega completamente el impacto el lenguaje. De aquí que los planteamientos de Lakoff y Johnson -expuestos en su libro Metáforas de la vida cotidiana - se aproximen de manera más lúcida a las implicaciones que el lenguaje tiene en la percepción, tanto dentro de la ficción de Villeneuve como en la vida cotidiana de los seres humanos.

\section{Lakoff, Johnson y Villeneuve: La Llegada desde un análisis de la metáfora}

El elemento que más destaca del trabajo de Lakoff y Johnson es el estudio de la metáfora como elemento de la percepción y comprensión humanas. Para estos autores, es un error concebir la metáfora como una figura literaria que atiende simplemente a factores estéticos. Para ellos, la metáfora tiene importantes implicaciones en la manera como los hablantes estructuran su percepción y la relación que tienen con su realidad, hasta el punto de que ciertas referencias conceptuales serían indescriptibles sin la ayuda de las metáforas. Dicho en palabras de Lakoff y Johnson: "si estamos en lo cierto al sugerir que nuestro sistema conceptual es en gran medida metafórico, la manera en que pensamos, lo que experimentamos y lo que hacemos cada día también es en gran medida cosa de metáforas" (Lakoff y Johnson, 2017, p. 35).

La mayor dificultad, advierten estos autores, radica en superar la larga tradición que desde Aristóteles ha considerado la metáfora como una suerte de "error permisible", una ilusión del lenguaje o un giro meramente estético. Estos antecedentes dificultan que la metáfora sea considerada como un elemento que proporciona verdadero conocimiento o que se dude de su capacidad para orquestar la percepción.

La metáfora se define como un mapping, una relación entre dos conjuntos conceptuales donde una idea referida a una experiencia sensorial más fácil de aprehender lega una serie de propiedades a una segunda idea más abstracta, a fin de facilitar la comprensión de esta segunda idea (Blanco, 2017, p. 211). Los ejemplos más ilustrativos propuestos por los autores son "el tiempo es dinero", "una discusión es una guerra" o "la mente es una máquina” (Lakoff y Johnson, 2017, pp. 36, 40, 61). 
Para su consideración de la metáfora, Lakoff y Johnson parten de las ideas de Whorf, pero las actualizan, considerando los factores fisiológicos y ambientales donde se desarrolla la vida de los seres humanos. Para estos autores, la metáfora no es únicamente una convención arbitraria: Lakoff y Johnson consideran que las metáforas responden a la estructura corporal de los hablantes y a la forma como se relacionan con su entorno. Estos aspectos fisiológicos también son considerados en la película de Villeneuve, donde las estructuras del lenguaje y el cuerpo de los extraterrestres posee una cierta correspondencia.

En la película, los personajes extraterrestres se definen por no poseer una forma humanoide: están compuestos por un tronco del que emergen siete extremidades circundantes. Es por eso que son bautizados como heptápodos. Adicionalmente, en el cuento que dio origen a la película, los heptápodos poseen una corona de ojos que les proporciona una visión de trescientos sesenta grados. Esto les permite mirar hacia todas las direcciones por lo que expresiones como adelante o atrás resultan absurdas. Esta correspondencia entre su cuerpo y su lenguaje permite asumir que para los heptápodos las nociones de principio y final carecen de sentido.

Lakoff y Johnson trabajan las nociones de orientación espacial y aseguran que "surgen del hecho de que tenemos cuerpos de un tipo determinado y que funcionan como funciona nuestro medio físico" (Lakoff y Johnson, 2017, p. 46). Esto no merma el impacto del lenguaje, sino que destaca los factores fisiológicos relacionados con la constitución de las lenguas. La representación de los heptápodos es un ejemplo de cómo una forma física diametralmente diferente a la humana da origen a un lenguaje igualmente disímil. La ficción permite considerar lo que sucede con criaturas parlantes tan distintas a los humanos y así evaluar la relación que ambos aspectos - cuerpo y lenguaje- poseen.

Para Lakoff y Johnson, esto opera así en las lenguas humanas, donde muchas de las referencias y conceptualizaciones metafóricas se corresponden con las proporciones del cuerpo humano. La tesis de estos autores da cuenta de una correspondencia semántica entre, por ejemplo, arriba y bueno: en tanto ser bípedo, el hombre está vivo y activo cuando se encuentra erguido. Caso contrario es el reposo o la muerte que se asocia con la imagen del cuerpo que yace o que se encuentra contra el suelo. Entonces arriba es vivo, y por tanto bueno, y abajo es 
muerto, o desfavorable. Johnson asegura que existe una importante implicación entre los contenidos conceptuales y los esquemas que surgen de la experiencia corporal (Johnson, 1991, p. 54). Es por esto que, como en el caso de los heptápodos, se puede concluir que el lenguaje humano sería distinto si la constitución de su cuerpo también lo fuese.

Otro elemento central de la teoría de Lakoff y Johnson que se ilustra en la película es la importancia de las distintas metáforas implícitas en el aprendizaje de una lengua. Para estos autores, así como en el film, la relación de los hablantes con el mundo está formada por estructuras de comportamiento cuyo origen conceptual está establecido por referentes metafóricos. En la historia de la doctora Banks, esto se hace explícito cuando ella descubre que los lingüistas de China se han comunicado con los extraterrestres a través del juego de mahjong.

Dentro de la ficción, el problema se suscita porque China ha movilizado sus tropas y parece disponerse para atacar a los extraterrestres, lo que supone un riesgo para el resto de los países. Al investigar este asunto, Louise descubre que sus homólogos chinos han enseñado a los heptápodos que cada conversación es una partida de mahjong con lo que muchos de los atributos del juego se trasladan, de manera implícita, como características de la conversación. El principal inconveniente es que en el juego ambos participantes aspiran a ser el ganador. Entender el diálogo como un juego de enfrentamiento implica asumir que en la conversación se pretende sobrepasar al adversario para hacerse con la victoria, con lo que la forma de comportarse y la dinámica a seguir se modifican. Las implicaciones tácitas que se derivan de esta noción de enfrentamiento proporciona el sustento de actitudes o acciones de los heptápodos que pueden ser desconcertante a los humanos. Es por esto que el grupo chino, sin haber notado dichas implicaciones, asume como un comportamiento bélico una reacción más acorde con la competición que con la charla.

El entender una conversación en términos de un juego con un ganador y un perdedor presenta como consecuencia una disposición diferente dentro del plano lingüístico y metalingüístico por parte de los participantes. Esto hace posible que un comportamiento coherente desde una perspectiva sea erróneo dentro de otra. Entonces es lícito concluir que, dentro de la ficción de La Llegada, la ambigüedad entre los conceptos de herramienta y arma que se da hacia el final de la película 
tiene su origen en el establecimiento de este marco referencial inicial. Cuando uno de los heptápodos le dice a Louise que su misión en la Tierra es ofrecer armas, esto genera mucha conmoción. La protagonista insiste en que es pertinente asegurarse de que los heptápodos comprenden la diferencia entre un arma y una herramienta, pues, al igual que en el caso del juego, un contexto conceptual distinto puede conectar el mismo referente con cualquiera de ambas descripciones. Otra opción que podría solventar la confusión sería asumir que arma y herramienta son utilizados como sinónimos en el lenguaje de los extraterrestres.

Desde la perspectiva antes expuesta es posible señalar dos explicaciones, sin que sean mutuamente excluyentes: primero, el empleo de la palabra arma puede estar condicionada por el marco conceptual de enfrentamiento que se deriva de la relación conversación-juego; y, segundo, la dificultad presente en la traducción desde el lenguaje heptápodo al inglés.

El primer caso se refiere a la relación que cada construcción lingüística tiene con su contexto físico y conceptual. Se asemeja mucho al ejemplo utilizado por Lakoff y Johnson donde destacan las diferencias entre la metáfora "una discusión es una guerra” - ampliamente difundida- y "una discusión es una danza” (Lakoff y Johnson, 2017, p. 36-37). Para estos autores, la significación de expresiones como defender una postura o atacar los argumentos son consecuencia de asumir las características de la primera metáfora para definir una discusión. Por otro lado, señalan los autores, la similitud con la danza podría aportar características colaborativas y nociones armónicas al concepto de discusión. Así, antes que un enfrentamiento, podríamos hablar de la discusión como un trabajo en equipo.

$\mathrm{Al}$ igual que para Lakoff y Johnson, la protagonista de la película se concentra en descifrar la actuación de los hablantes inmersos en ciertos parámetros conceptuales. No se trata únicamente del aprendizaje de los códigos concretos y sus relaciones; sino de sus implicaciones con respecto al desenvolvimiento de los hablantes con su entorno. "Hablamos de discusiones de esa manera porque las concebimos de esa manera y actuamos según la forma en que concebimos las cosas" (Lakoff y Johnson, 2017, p. 38). En el contexto discusión-guerra o discusión-juego la dinámica competitiva impera. Al no compartir la metáfora 
primaria, la comunicación entre heptápodos y humanos se vuelve confusa y da lugar a los malentendidos.

El segundo aspecto hace referencia directa a las dificultades de la traducción. Con esto no se pretende regresar a la noción perteneciente al relativismo lingüístico más radical que entiende la traducción como una quimera; sino rescatar la idea de particularidad de cada lengua y la contingencia de sus relaciones. Las traducciones pueden ser equívocas porque, como en la metáfora, existe una negociación de significado que considera los matices presentes en cada idioma.

Así como se refleja en la ficción, para las lenguas humanas la comprensión metafórica no es accesoria, sino que constituye una parte medular de la dinámica de los hablantes con su entorno. Al constituir el significado, las metáforas originan dinámicas de sentido propias de ciertos contextos. Las acciones son acordes, desproporcionadas o confusas en función de estas metáforas primarias, por lo que su estudio es sustancial.

Aceptar el impacto de la metáfora en las formas de percepción, implica el abandono de una postura objetivista, incompatible con su carácter mutable. Con respecto a esto, Lakoff y Johnson (2017) rechazan directamente la existencia de una verdad absoluta, universal e incondicional, entendiendo que las características intersubjetivas y maleables propias de la metáfora tienen una injerencia directa en la comprensión de esta verdad. Al aprehender el mundo con una cierta correspondencia con las estructuras metafóricas y gramaticales del lenguaje, ya no es posible entender el conocimiento como algo preexistente que pasa a formar parte de la realidad de unos humanos pasivos y receptivos. Así mismo, la verdad estará relacionada con las estructuras conceptuales y lingüísticas a través de las cuales se trata de acceder a ella. Estas afirmaciones no implican una devaluación de la verdad, sino que pretenden destacar su componente humano, su relación con las prácticas y objetivos más vitales de los hablantes. Al igual que las nociones de tiempo o espacio, que están dotadas de una dimensión lingüística y social, sucede con conceptos metafísicos como la verdad. La constitución física predeterminada y el lenguaje como marco de referencia ajustable proporcionan a los hablantes la simultánea consistencia y flexibilidad con la cual comprenden su realidad. 
A pesar de lo afirmado, su propiedad cambiante no implica arbitrariedad o inconsistencia, dentro de la metáfora es posible el error, de donde se originan fenómenos como la ambigüedad. Este es el caso - ahora atendiendo a su estructura narrativa y no al contenido de su historia- de La Llegada.

\section{La experiencia metafórica del espectador}

Como ya se ha expresado, Lakoff y Johnson destacan el carácter configurador de la metáfora, siendo constructora de sentido y de definición. Es por eso que nociones que podrían entenderse como intuitivas están realmente muy marcadas por estructuras culturales, propias de cada lengua.

El caso del tiempo resulta especialmente controvertido, pues parece que cada cultura tiene formas convencionales de referirse a él (Blanco, 2017, p. 177). A pesar de la dificultad para definirlo de forma universal, los seres humanos lo han convertido en un elemento fundamental de su cotidianidad. La metáfora más común, como se ve reflejado en los ejemplos de Lakoff y Johnson, consiste en ubicar espacialmente los momentos temporales. Esta referencia está fusionada con la definición de "tiempo" de tal manera que resulta imposible dar cuenta de su concepto sin aludir a su referente metafórico.

En las lenguas occidentales la estructura temporal está vectorizada, lo que la hace intrínsecamente direccional. En un plano horizontal: el pasado está a la izquierda y el futuro, a la derecha. En un plano tridimensional con referencia en el ego hablante: el pasado está detrás del sujeto y el futuro está frente a él. Estas ideas se han incorporado al pensamiento como estructuras base, por lo que los hablantes tienden a pensar que tales nociones trascienden sus propias formas lingüísticas.

Pensar el futuro como un lugar que se encuentra delante del sujeto y que se aproxima a él implica asumir otra metáfora primaria: el tiempo es un espacio que permite el movimiento. Podría considerarse que es la persona quien se aproxima al futuro o que, por el contrario, que la persona permanece quieta y el futuro es el que se mueve hacia ella. En cualquier caso, se establece una relación de proximidad donde los acontecimientos del pasado se dejan atrás, quedan en la espalda, y los hechos futuros se reciben en tanto el movimiento tiende a ellos. 
Por otro lado, hay lenguas donde se asume que el futuro está a las espaldas del sujeto (como los hablantes de la lengua aymara de Sudamérica) y que aceptan otra metáfora primaria: el conocimiento es visión. Debido a que el futuro no ha acaecido, no se puede tener ninguna certeza sobre sus características más allá de meras especulaciones. En contraparte, del pasado es posible hacer un relato específico. El pasado es conocido, mientras que el futuro es solo suposición. Desde la metáfora el conocimiento es visión es lógico concluir que el pasado debe presentarse frente al sujeto, para que este sea capaz de verlo y dar cuenta de él. La visión unidireccional humana implica que la capacidad de visión, y por tanto de conocer a través de ella, refiere solo aquello que está adelante. Del mismo modo es posible concluir que el futuro debe estar a las espaldas del hablante, donde no pueda ser visto y su referencia es imprecisa.

Ambas metáforas tienen un importante carácter fisiológico que refiere de manera directa a la constitución del cuerpo humano. La visión, como sentido principal, es capaz de dar cuenta de aquello que entra dentro de su rango de alcance. Igualmente, la metáfora del movimiento también está fuertemente ligada a esta característica. El movimiento natural es hacia adelante y esto, a su vez, está condicionado por la posición de los ojos en la cabeza.

El grado de injerencia que estas estructuras tienen en la percepción de nociones como el tiempo es tan grande que la literatura y el cine han logrado fusionar las dos visiones antes expresadas y han creado los flashbacks y la analepsis. Este recurso es el que da sentido a la narrativa utilizada por Villeneuve en La Llegada. Aun cuando la noción lineal del tiempo resulta la más conocida, la idea de flashback no es del todo extraña. La relación constante con ficciones - literarias y cinematográficas - que emplea este recurso habitúa a los hablantes a concebir con naturalidad estos saltos temporales que rompen la progresión de un relato, que narra de forma intermitente hechos del pasado y del presente. A pesar de su cotidianidad, no deja de ser llamativo lo incongruente que puede resultar esta noción.

Un flashback no solo implica ubicar el pasado delante del sujeto (idea que, como se ha señalado, es natural para algunas culturas), sino que lo relevante del flashback se centra en que asume la idea del pasado como algo que se deja atrás, pero que es capaz de reaparecer en la progresión hacia el futuro, bloqueando la 
visión, al aparecer delante del sujeto con la representación de un recuerdo. El flashback destaca como recurso no tanto porque suponga que el pasado puede presentarse frente a los ojos sino por la suposición de que puede interrumpir el recorrido hacia el futuro. La simplicidad de la concepción lineal del tiempo se ve matizada por la aparición momentánea de sucesos no cronológicos que sugieren un entramado más complejo que no asume solamente que el tiempo transcurre de un extremo a otro.

El concepto de tiempo, como muchos otros, revela su lado más flexible, sustentado en las relaciones metafóricas propias de la narración. Posiblemente, la metáfora que más se ajuste al concepto de flashback sea la memoria es visión (noción mucho más afín a la metáfora del conocimiento como visión). Con base en esta idea del recuerdo como interrupción, se presenta el relato de la película La Llegada. Uno de los tópicos centrales del film consiste en la representación y concepción del tiempo, por lo que la propia narración del largometraje se convierte en una experiencia de esta distorsión. "La expresión del movimiento (movimiento como cambio, sinónimo de crisis, de conflicto o drama en el discurso) constituye la esencia del cine, pero también lo es la anulación del mismo" (Ustarroz, 2011, p. 43). Esto es posible si se presupone el manejo del flashback como se ha explicado anteriormente. Solo de esta forma resulta sorpresivo el giro final que Villeneuve da al relato.

El film se apoya en esta concepción del flashback para engañar al espectador y hacerle creer que está viendo el pasado de la protagonista cuando no es así. Se presupone que las narraciones -y el tiempo en general- se manejan bajo una noción vectorizada, pero donde la idea de flashback es tan natural que no presenta ningún cuestionamiento. El espectador es capaz de seguir la historia a pesar de que la secuencia implique pausar los relatos del presente para contar otros episodios - cada uno con estructura lineal interna. Es por eso que en la presentación de un relato discontinuo, las imágenes que no pertenecen al presente se asumen como hechos del pasado. La película maneja esto para presentar un final sorprendente donde el espectador reconoce que todos esos episodios son realmente hechos del futuro de la protagonista. El uso de esta forma narrativa es inesperada, aun cuando la metáfora que ubica el futuro delante del sujeto parezca más congruente con la noción del "futuro como un objeto al 
alcance de la vista”. Esta última referencia tiene mayor afinidad con la metáfora "el conocimiento es visión” tomada como premisa.

La Llegada no solo narra una historia que interpela la noción espacial y temporal que las lenguas humanas - especialmente los occidentales - poseen, sino que ella misma se cuenta a través de esta concepción, convirtiéndose en un ejemplo de lo que trata de presentar con sus protagonistas. En la película, el final es lo primero que se muestra, dando el sentido de palíndromo a todo el relato. Para Louise, el futuro se mezcla con el presente; para el espectador, el futuro se confunde con el pasado; y en la historia como conjunto se construye una percepción temporal a modo de red, donde los momentos se conectan unos con otros de forma no lineal.

Si ha de demostrarse que los lenguajes tienen una importante injerencia en el pensamiento, la experiencia de esta película debe ser un ejemplo, por su historia y por el manejo de lo que el espectador entiende como pasado y futuro. Mirar La Llegada es, quizás, lo más cercano que puede experimentarse al uso del lenguaje heptápodo, enfocando la atención en la importancia conceptual de las metáforas en las que los hablantes viven.

\section{Conclusiones}

Así como la literatura, a lo largo de los años el cine se ha convertido en plataforma de expresión y desarrollo para diversos temas de la filosofía. Múltiples historias, apoyadas en las licencias que concede la ficción, han empleado este formato para cuestionar diversos temas y suscitar la reflexión. En ocasiones, el cine es capaz de ensayar una respuesta o conclusión; en otros casos, trabaja sobre los planteamientos, delimitando y precisando las preguntas aún vigentes. De este modo "La ciencia ficción nos proporciona un laboratorio de experimentos mentales en el que tanto el escritor como el lector pueden probar cosas con amplitud y observar qué es lo que pasa” (Hagen, citado en Blanco, 2017, p. 54).

La Llegada se considera como uno de esos casos en los que su trama o su construcción narrativa llama al asombro. Su planteamiento principal radica en las implicaciones que las estructuras del lenguaje tienen en la percepción, es decir, cuestiona si el idioma que maneja cada persona se limita a un medio de 
expresión de realidades preexistentes o si, por el contrario, estas estructuras moldean en cierta medida aquello que refieren.

Este es el mismo cuestionamiento que guía el trabajo de Lakoff y Johnson, en defensa de la metáfora. La comprensión de la realidad sobre la cual los hablantes desarrollan su vida cotidiana está armada sobre una red de relaciones metafóricas implícita en cada construcción lingüística. La metáfora, y el lenguaje en general, no se refieren a simple descripción o referencia de un mundo inamovible, sino que se conciben bajo los efectos experimentados por Louise: como estructuras de percepción, pensamiento y acción.

El relato de la doctora Banks, tanto en su contenido como en su forma, es una clara representación de cómo las lenguas y sus usos constituyen parte indispensable de la realidad humana. Las suposiciones implícitas es que cada relación conceptual configura la forma como se organiza el mundo y, sobre esa base, se toman las decisiones con respecto al modo de obrar. Las ideas más fundamentales - el tiempo, el espacio, la memoria, la identidad - se presentan como estructuras sostenidas sobre esquemas lingüísticos, donde modificaciones conceptuales afectan también las formas de percepción. Las acciones de los humanos se desarrollan bajo este esquema flexible y abierto a la contingencia de los usos. Será necesario renunciar al mito objetivista y subjetivista, pensar la metáfora como una estructura transversal y comunitaria, y entender el lenguaje como una forma de vida.

\section{Referencias bibliográficas}

Blanco, A. (2017). La relatividad lingûistica (variaciones filosóficas). Madrid: Ediciones Akal.

Chiang, T. (2015). La historia de tu vida. Madrid: Alamut.

Conesa, F. \& Nubiola, J (1999). Filosofia del lenguaje. Barcelona: Herder.

Ibañez, T (2001). Municiones para disidentes. Realidad-Verdad-Politica. Barcelona: Gedisa.

Jiménez, R. (2015). Cuando el horizonte es el presente. El tiempo en suspensión en la obra de Chien-Chi Chang. Fotocinema. Revista científica de cine y fotografia, $11, \quad$ pp. 170-188. Disponible: http://www.revistafotocinema.com/

Johnson, M. (1991). El cuerpo en la mente. Madrid: Debate. 
Lakoff, G. \& Johnson, M (2017). Metáforas de la vida cotidiana. Madrid: Cátedra.

Ustarroz, C. (2011). La suspensión del momentum cinematográfico. L’Atalante. Revista de Estudios Cinematográficos, 12, pp. 42-47. Disponible: http://www.revistaatalante.com/index.php?journal=atalante\&page=issu e\&op=view\&path\%5B\%5D=7\&path\%5B\%5D=showToc

Villeneuve, D (2016). Arrival. Estados Unidos: Lava Bear Films / FilmNation Entertainment / 21 Laps Entertainment.

Whorf, B. (1971). Lenguaje, pensamiento y realidad. Barcelona: Barral. 